\title{
Chrysanthemum Boom
}

\author{
Karuna Tamrakar Karki ${ }^{1}$ \\ ${ }^{1}$ Department of Neurosurgery, B and C Medical College Teaching Hospital and Research Center, Birtamode, \\ Jhapa, Nepal. \\ Correspondence: \\ Dr Karuna Tamrakar Karki \\ Department of Neurosurgery, B \& C Medical College and Teaching Hospital \& Research \\ Center,Birtamode,Jhapa,Nepal. \\ Email: tamrakarkaruna@gmail.com \\ Phone: +9779843405124
}

$\mathrm{S}$ elective intra-arterial angiography is crucial to determine the vascular supply to the tumor in detail. Meningiomas are highly vascular brain tumors which may need massive amount of blood transfusion during surgical resection. Embolization of dural base tumors has emerged as a pre operative adjuvant therapy. Angiographic features of meningioma includes: external carotids artery feeders, spoke wheel appearance, mother in law sign and sunburst appearance (Figure 1). All these angiographic features demonstrate the chrysanthemum boom effect during selective digital subtraction angiography.
Although MR angiography has decreased the potential need of endovascular angiography, the latter remains the gold standard technique to classify the tumor that is subsequently helpful for planning surgery with or without preoperative embolization. Hence catheter angiography and endovascular selective intra-arterial embolization is indispensible to reduce intraoperative blood loss. This ultimately gives total tumor resection in short time.
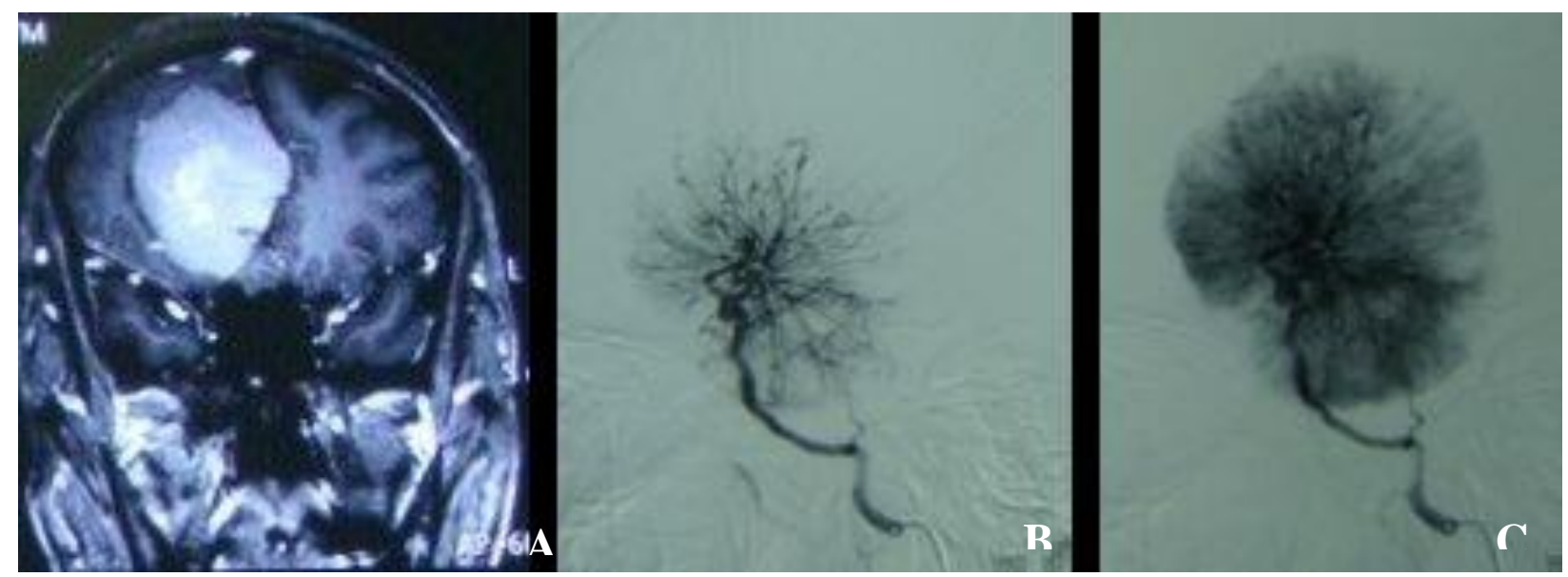

Figure 1: Chrysanthemum boom effect in selective ECA arteriography. A: Parasagittal Meningioma with homogenous contrast enhancement in MRI. B: selective digital subtraction angiography in arterial phase (spoke wheel appearance). C: $\begin{array}{lllllll}\text { venous } & \text { phase: } & \text { Chrysanthemum } & \text { booming }\end{array}$

resection time. Arterial supply is typically identified by diffuse area of parenchymal staining in DSA. This is especially practical for tentorial base or skull base tumors with abundant blood

65

Date submitted: $12 / 10 / 2019$

Date accepted: 8/12/2019 supply either unilateral or bilateral from the external carotid artery. Embolization therapy involves selective intra-arterial catheterization of main feeder and subsequent injection of micro

egneuro Volume 02, Issue 01, 2020 DOI: https://doi.org10.3126/egn.v2i1.27474 
particles like polyvinyl alcohol (PVA). ${ }^{1}$ Preoperative tumor embolization is not always appropriate. Complete angiographic assessment (6-vessel intra-arterial angiography) of all major supply to the head and neck is very crucial to avoid inadvertent embolization of major ICA branches. Number of cases have been reported with neurological deterioration after tumor embolization. $^{2}$ Dangerous anastomosis between internal carotid and external carotid arteries has to be thoroughly understood before stepping into tumor embolization. ${ }^{3}$ Besides anonymous anastomosis, improperly identified vascular territories, reflux of embolic particles, tumor rebleed and swelling are the major drawback of preoperative tumor

\section{References}

1. Karki KT, Koirala S. An Unusual Variant of Cerebellopontine Angle Schwannoma in a Young Nepalese Girl. J Clin Exp Pathol. 2017;7(307):2161-0681.

2. Bendszus M, Monoranu CM, Schütz A, Nölte I, Vince GH, Solymosi L. Neurologic complications after particle embolization of intracranial meningiomas. American Journal of Neuroradiology. 2005 Jun 1;26(6):1413-9.

3. Geibprasert S, Pongpech S, Armstrong D, Krings $\mathrm{T}$. Dangerous extracranial-intracranial anastomoses and supply to the cranial nerves: vessels the neurointerventionalist needs to know. American journal of neuroradiology. 2009 Sep 1;30(8):1459-68. 